\title{
Inhibition of Mammalian Target of Rapamycin Signaling with Rapamycin Prevents Trauma-Induced Heterotopic Ossification
}

\author{
Ammar T. Qureshi, ${ }^{*}$ Devaveena Dey, ${ }^{*}$ Erin M. Sanders, ${ }^{*}$ Jonathan G. Seavey, ${ }^{* \dagger}$ Allison M. Tomasino, ${ }^{*}$ Kaitlyn Moss, \\ Benjamin Wheatley, ${ }^{* \dagger}$ David Cholok, Shawn Loder, ${ }^{\ddagger}$ John Li, ${ }^{\ddagger}$ Benjamin Levi, ${ }^{\ddagger}$ and Thomas A. Davis ${ }^{*}$
}

From the Regenerative Medicine Department, * Naval Medical Research Center, Silver Spring, Maryland; the Department of Surgery, ${ }^{\ddagger}$ University of Michigan Health Systems, Ann Arbor, Michigan; and the Department of Surgery, ${ }^{\dagger}$ Uniformed Services University and the Walter Reed National Military Medical Center, Bethesda, Maryland

Accepted for publication July 26, 2017.

Address correspondence to Thomas A. Davis, Ph.D., Department of Surgery, Uniformed Services University and the Walter Reed National Military Medical Center, 4301 Jones Bridge Rd., Bethesda, MD 20814. E-mail: thomas. davis@usuhs.edu.

\begin{abstract}
A pressing clinical need exists for $63 \%$ to $65 \%$ of combat-wounded service members and $11 \%$ to $20 \%$ of civilians who develop heterotopic ossification ( $\mathrm{HO}$ ) after blast-related extremity injury and traumatic injuries, respectively. The mammalian target of rapamycin pathway is a central cellular sensor of injury. We evaluated the prophylactic effects of rapamycin, a selective inhibitor of mammalian target of rapamycin signaling, on $\mathrm{HO}$ formation in a rat model of blast-related, polytraumatic extremity injury. Rapamycin was administered intraperitoneally daily for 14 days at $0.5 \mathrm{mg} / \mathrm{kg}$ or $2.5 \mathrm{mg} / \mathrm{kg}$. Ectopic bone formation was monitored by micro-computed tomography and confirmed by histologic examination. Connective tissue progenitor cells, platelet-derived growth factor receptor- $\alpha$-positive cells, and $\alpha$-smooth muscle actin-positive blood vessels were assayed at postoperative day 7 by colony formation and immunofluorescence. Early gene expression changes were determined by low-density microarray. There was significant attenuation of 1) total new bone and soft tissue ectopic bone with $0.5 \mathrm{mg} / \mathrm{kg}$ $(38.5 \%$ and $14.7 \%)$ and $2.5 \mathrm{mg} / \mathrm{kg}$ rapamycin $(90.3 \%$ and $82.9 \%)$, respectively, 2 ) connective tissue progenitor cells, 3) platelet-derived growth factor receptor- $\alpha$-positive cells, 4$) \alpha$-smooth muscle actin-positive blood vessels, and 5) of key extracellular matrix remodeling (CD44, Col1a1, integrins), osteogenesis (Sp7, Runx2, Bmp2), inflammation ( $C x c 15,10, I L 6, C c l 2)$, and angiogenesis (Angpt2) genes. No wound healing complications were noted. Our data demonstrate the efficacy of rapamycin in inhibiting blast trauma-induced $\mathrm{HO}$ by a multipronged mechanism. (Am J Pathol 2017, 187: 2536-2545; http://dx.doi.org/10.1016/j.ajpath.2017.07.010)
\end{abstract}

A common complication of military service members who sustain severe battlefield blast-related extremity injury is the extra-skeletal development of mature endochondral bone within damaged/healing soft tissue, resulting in heterotopic ossification (HO) in approximately $63 \%$ to $65 \%$ of blastrelated extremity amputations and nearly $62 \%$ of limbsparing procedures. ${ }^{1-3}$ Surgical excision of mature HO still remains the standard of care in the combat-injured and

\footnotetext{
Supported in part by Congressionally-Directed Medical Research Programs (CDMRP) grant W81XWH-16-2-0051 (T.A.D.), NIH/National Institute of General Medical Sciences grant K08GM109105-0 (B.L.), the Plastic Surgery Foundation (B.L.), an Association for Academic Surgery Roslyn Award (B.L.), an American Association for the Surgery of Trauma Research \& Education Foundation Scholarship (B.L.), an American Association of Plastic Surgery Academic Scholarship (B.L.), an American College of Surgeons Clowes Award (B.L.), a Plastic Surgery Foundation (PSF) and the American Association of Plastic Surgeons (AAPS) Pilot
}

Award (B.L.), and the International Fibrodysplasia Ossificans Progressiva Association (B.L.).

Disclosures: None declared.

The opinions or assertions contained in this paper are the private views of the authors and are not to be construed as reflecting the views, policy, or positions of the Department of the Navy, Department of Defense, the Uniformed Services University of the Health Sciences, or any other agency of the U.S. government. 
amputee population and can be wrought with complications, including further delay in rehabilitation and, in many cases, recurrence of HO., ${ }^{4,5}$ Consequently, there is an unmet clinical need for a safe and effective prevention for blast trauma-induced HO.

Traumatic HO, as seen in the blast-injured population, is also common in the civilian setting, occurring as a profound sequela of severe burns, crush injury, traumatic brain and spinal cord injuries, and arthroplasties. The common underlying trigger for all forms of traumatic $\mathrm{HO}$ is acute inflammation and extensive damage of soft tissue, with its associated vasculature and innervations. Infection also increases the risk of HO. ${ }^{6,7}$ The simultaneous activation of inflammatory, wound healing, and tissue repair pathways culminates in the deposition of a cartilaginous anlage, by a yet unknown mechanism, initiating the phenomenon of endochondral ossification. ${ }^{8,9}$ Recently, we developed a rat trauma-induced HO model that involves blast-related limb injury, femoral fracture, quadriceps crush injury, amputation, and infection with methicillin-resistant Staphylococcus aureus (MRSA) from combat wounds. ${ }^{5,10}$ With the use of this model, we have determined that critical molecular changes occur early after trauma, leading to ectopic bone growth, ${ }^{8}$ and this timing is key for defining the therapeutic window for safe and effective prevention strategies.

The mammalian target of rapamycin (mTOR) signaling pathway is a central regulator of cellular processes, such as cell survival, metabolism, proliferation, differentiation, and senescence. ${ }^{11}$ The mTOR pathway regulates these processes by integrating extracellular signals with changes in intracellular signal transduction. Some of the extracellular signals shown to activate this pathway, leading to anabolic changes, include glucose and amino acid levels, hypoxia, and growth factors. Inflammatory molecules (cytokines and chemokines) inhibit this pathway, leading to catabolic changes, typically autophagy. mTOR is a serine-threonine kinase, functioning by two distinct multi-protein complexes, the rapamycin-sensitive mTORC1 and the rapamycin-insensitive mTORC2. Rapamycin, a bacterial macrolide, binds to FK506-binding protein, and by the FK506-binding protein/mTOR binding site, it inhibits the mTORC1 complex. This leads to inhibition of the anabolic processes of protein and lipid synthesis, mitochondrial function, and activation of catabolic processes, such as autophagy.

Given the involvement of the mTOR pathway as a sensor and effector of inflammation, hypoxia, and nutrient changes, ${ }^{12}$ all of which are significantly altered in traumatic injuries, we hypothesized that inhibiting this pathway might block development of trauma-induced HO. The classic inhibitor of the mTOR pathway, rapamycin (sirolimus) is a potent Food and Drug Administration (FDA)-approved immunosuppressant, typically used for the prevention of kidney allograft rejection. ${ }^{13}$ Rapamycin has been shown to inhibit cell cycle progression in alloreactive T cells, ${ }^{14}$ inhibit kinase activity associated with lymphocyte proliferation, ${ }^{15}$ attenuate stem/progenitor cell mobilization, and suppress chondro-angio-osteogenic activity associated with endochondral bone development. ${ }^{10,16}$

The aim of this study was to assess the effect of systemic mTOR pathway inhibition on heterotopic bone formation in vivo by administering rapamycin for a short period after injury. We therefore sought to determine the efficacy of rapamycin in preventing ectopic bone formation at sites of early HO by assessing i) soft tissue-associated chondrogenesis, osteogenesis, and angiogenesis; ii) suppression of tissue osteoprogenitor cell activity; iii) changes in expression of genes involved in ectopic endochondral ossification; and, finally, iv) the frequency and types of complications associated with this treatment. In addition, we wanted to ensure that rapamycin use would not increase risk of wound breakdown or infection.

\section{Materials and Methods}

\section{Animals}

A total of 71 young adult pathogen-free male SpragueDawley rats (Rattus norvegicus; 400 to $600 \mathrm{~g}$ ) were purchased from Taconic Farms (Germantown, NY). All animals were housed in clean plastic cages and kept on a 12-hour light/dark cycle with unlimited access to food (standard rodent chow) and fresh water ad libitum. The study protocol (15-OUMD-19S) was reviewed and approved by the Walter Reed Army Institute of Research/ Naval Medical Research Center Institutional Animal Care and Use Committee in compliance with all applicable federal regulations governing the protection of animals in research.

\section{Trauma-Induced Blast and Extremity Injury}

Rats $(n=55)$ were anesthetized with $2 \%$ to $4 \%$ isoflurane and then exposed to blast overpressure $(120 \pm 7 \mathrm{kPa})$ by a pneumatically driven shock tube, followed by controlled femoral fracture, a 1-minute soft tissue quadriceps crush injury, limb amputation through the zone of injury, and inoculation of the wound with $1 \times 10^{6}$ colony-forming units of MRSA as previously described. ${ }^{7,17}$ For experiments involving ex vivo cell enrichment and culturing, MRSA inoculation was omitted ( $n=8$ rats). Appropriate debridement of devitalized tissue was performed to remove any comminuted bone fragment, followed by hamstring and quadriceps myoplasty over the exposed residual femur. After operation, a dose of sustained release buprenorphine $(1.2 \mathrm{mg} / \mathrm{kg})$ was administered subcutaneously on day of operation and 48 to 72 hours after operation for pain or distress. Daily observational wound healing assessments of the amputation sites (ASs) were made and documented for evidence of infection and wound-healing complications. 


\section{Rapamycin Administration and Treatment Conditions}

Starting at postoperative day (POD) 1 , rats were given daily intraperitoneal injections $(0.5 \mathrm{mg} / \mathrm{kg}$ or $2.5 \mathrm{mg} / \mathrm{kg})$ of freshly prepared rapamycin (sirolimus; Cayman Chemical, Ann Arbor, MI) in $100 \mu \mathrm{L}$ of $3 \%$ dimethyl sulfoxide/saline used as diluent (vehicle control) for 14 days. In study 1, a longitudinal observation study, the long-term efficacy of rapamycin was evaluated on ectopic bone attenuation and possibly rebound effect after drug treatment cessation. Rats were randomly divided into three treatment groups. Group I received vehicle control $(n=6)$, group II received rapamycin at $0.5 \mathrm{mg} / \mathrm{kg}(n=10)$, and group III received rapamycin at $2.5 \mathrm{mg} / \mathrm{kg}(n=7)$. Wound sites for impaired tissue integrity and animal health status were monitored daily, and body weight was recorded biweekly. Total new bone volume was determined by micro-computed tomography at 1,2 , and 3 months after injury. Animals were euthanized on POD 84 . In study 2 , the effects of mTOR signaling inhibition at POD $7(2.5 \mathrm{mg} / \mathrm{kg}$ per day for 7 days; POD 1 through POD 7) were comparatively assessed on the development of osteogenic connective tissue progenitor (CTP-O) cells, platelet-derived growth factor receptor $(\mathrm{PDGFR}) \alpha^{+}$mesenchymal stromal cells, and $\alpha$-smooth muscle actin $(\alpha \text {-SMA })^{+}$blood vessels in the soft tissue surrounding the AS in non-MRSA-infected rats treated $(n=8)$. Muscle tissue collected from uninjured agematched rats $(n=4)$ served as normalization controls.

\section{Quantitative Micro-Computed Tomography Analysis}

Progression of ectopic bone development at the site of amputation was assessed using a high-resolution microcomputed tomography system (SkyScan 1176; Bruker microCT, Kontich, Belgium) and a standard phantom for normalization. Total new bone (differential new bone from native bone) and soft tissue ectopic bone (not associated with residual femur) formation were calculated by two independent investigators (A.T.Q., J.G.S.) using Bruker microCT volumetric software version $1.14 .10 .0+$ as previously described. ${ }^{17}$

\section{Bone and Skeletal Muscle Histologic Analysis at the AS}

At POD 84 the residual amputated limb from each rat was surgically excised after euthanization. Tissues were fixed in phosphate-buffered formalin for at least 48 hours, decalcified in $3 \%$ formic acid, embedded in paraffin, serially sectioned $(5 \mu \mathrm{m})$, and stained with hematoxylin and eosin (Histoserv, Inc., Germantown, MD) for light microscopic examination by a pathologist blinded to the experimental groups. ${ }^{8}$

\section{Immunofluorescence Staining}

For immunofluorescence staining for PDGFR $\alpha$ and $\alpha$-SMA, paraffin-embedded section $(10 \mu \mathrm{m})$ of residual femur from amputated limbs of rapamycin-treated and vehicle control samples were deparaffinized and rehydrated in xylenes and graded ethanol. Antigen retrieval was performed with citrate solution $\mathrm{pH}$ 6. Samples were quenched for autofluorescence in 3\% glycine before blocking and permeabilization. Polyclonal goat anti-rat or mouse anti-rat primary antibodies (Santa Cruz Biotechnology, Santa Cruz, CA) were applied overnight at $4^{\circ} \mathrm{C}$. After washing, fluorescently conjugated secondary antibodies tagged with either Alexa Fluor 488 or Alexa Fluor 594 (Thermo Fisher Scientific, Waltham, MA) were applied. Nuclear counterstain was achieved using Hoechst 33342 (Life Technologies, Carlsbad, CA) and mounted with ProLong Gold Antifade Reagent (Life Technologies). All fluorescently stained images were taken using a Leica Upright SP5X Confocal Microscope (Leica, Wetzlar, Germany) or Olympus BX-51 upright light microscope (Olympus, Tokyo, Japan) equipped with standard DAPI, $488 \mathrm{~nm}$, and tetramethylrhodamine isothiocyanate cubes attached to an Olympus DP-70 high-resolution digital camera. Each site was imaged in all channels and overlaid in DPViewer version 03.03 (Olympus Corporation, Tokyo Japan) before examination in Photoshop CC 2015 (Adobe, San Jose, CA).

To quantify staining counts on five high-powered fields chosen at random were performed from across five distinct sections. Each high-powered field was hand counted, and results were tallied from three separate reviewers (D.C., S.L., and J.L.) to ensure consistency. Immunofluorescent staining was regarded as positive when red/green channel signal overlapped with or was immediately proximal to DAPI-positive staining in the blue channel.

\section{CTP-0 Colony-Forming Cell Assays and Analysis of ALP Activity of CTP-O Cells}

Biopsies of skeletal muscle were aseptically harvested from the zone of injury in rapamycin- and vehicle control-treated rats at POD 7 as previously described. ${ }^{4}$ Briefly, the samples were devoid of fascia and fat, weighed, thoroughly minced into slurry, incubated in a solution of $300 \mathrm{U} / \mathrm{mL}$ of collagenase type II (Worthington, Lakewood, NJ), reconstituted in phosphate-buffered saline (PBS) for 2 hours to release cells from intact fibers. A single-cell suspension was generated by consecutive straining through $70-\mu \mathrm{m}$ mesh, followed by $40-\mu \mathrm{m}$ nylon mesh cell strainers (BD BioSciences, San Jose, CA). Red blood cells were lyzed by incubating filtered cells in $5 \mathrm{~mL}$ of ACK lysis buffer (Lonza, Walkersville, MD) for 5 minutes, followed by washing cells with ice-cold PBS. The red blood cell-depleted, filtered nucleated cells were serially diluted and seeded in duplicates in two-well chamber slides (Sigma-Aldrich, St. Louis, MO) at a concentration of 1000 cells/well in either regular growth mesenchymal stromal cell medium (Dulbecco's modified Eagle's medium-F12, supplemented with $10 \%$ fetal bovine serum, $100 \mathrm{U} / \mathrm{mL}$ penicillin, and $100 \mu \mathrm{g}$ of streptomycin; 
Lonza) or changed to osteogenic differentiation medium (Lonza) after overnight incubation. Cells were incubated for 6 days in fully humidified atmosphere of $37^{\circ} \mathrm{C}$ containing $5 \% \mathrm{CO}_{2}$. For CTP colony-forming cell assay, chambers were washed with PBS, followed by fixation with $100 \%$ methanol for 3 to 5 minutes, air dried, stained with Crystal violet for 2 to 3 minutes and then washed five times with tap water. Phase contrast light microscope [Olympus; 1X73 inverted microscope equipped with cellSense Dimension software version 1.13 (Olympus Corp., Center Valley, PA)] was used to manually count distinct CTP-O colonies (aggregates $>20$ cells) as described previously. ${ }^{6}$ For quantification of cellular alkaline phosphatase (ALP) activity (ALP assay; SigmaAldrich), media was discarded, followed by a single wash of attached cells with PBS. Cells were lyzed in 150 $\mu \mathrm{L}$ of $1 \%$ Triton $\mathrm{X}-100$ for 1 to 2 minutes (on a shaker, room temperature), followed by the addition of an equal volume of p-nitrophenyl phosphate substrate (1 to 2 minutes on a shaker, room temperature). One hundred microliters per well of this solution was divided into three wells of a 96-well flat-bottomed plate, and absorbance was read immediately at $405 \mathrm{~nm}$ for 20 consecutive cycles. Absorbance values at cycles 10 and 15 were used for comparative analysis of ALP activity for all samples.

\section{RNA Isolation and Gene Expression}

Muscle tissue collected from the zone of injury in the amputated limb was placed and preserved in RNA-Later (Ambion, Inc., Austin, TX). Total mRNA was isolated using RNeasy Plus Universal kits (Qiagen, Valencia, CA). ${ }^{8}$ RNA was quantitated as previously described, and RT-PCR was used to convert $1 \mu \mathrm{g}$ of RNA to cDNA using iScript cDNA synthesis kit (Bio-Rad, Hercules, CA). ${ }^{7}$ Quantitative real-time PCR for 83 chondrogenic, angiogenic, and osteogenic gene targets was performed on a QuantStudio 7 Flex Real-Time PCR System (Applied Biosystems, Foster City, CA) using a custom-made PCR Array (Bio-Rad). ${ }^{7,8}$ All target genes were normalized to the reference housekeeping gene $G A P D H$. Relative gene transcript expression was calculated using the $2^{-\Delta \Delta \mathrm{Ct}}$ method.

\section{Statistical Analysis}

Noncontinuous gene expression data were analyzed with the $U$-test. Intraclass correlation coefficient was used to investigate inter-rater agreement between the investigator scores in calculating the volume of ectopic bone from microcomputed tomography images. The Welch's two-sample $t$-test and Fisher test were used to determine the statistical differences in ectopic bone volume $\left(\mathrm{mm}^{3}\right)$ between the rapamycin- and vehicle control-treated rats. All data are presented as means \pm SEM unless otherwise specified, and $P$ values $<0.05$ were considered to be statistically significant.

\section{Results}

After operation, all rats were completely ambulatory within 24 hours of injury and survived with no major adverse events with regard to wound dehiscence, wound failure, and weight loss. The volume of both total new bone (Figure 1A) and soft tissue ectopic bone (Figure 1B) at POD 84 were significantly less with rapamycin treatment compared with rats treated with vehicle control, respectively $[0.5 \mathrm{mg} / \mathrm{kg}$ rapamycin: $25.5 \pm 4.3 \mathrm{~mm}^{3}$ (38.5\% reduction, $P=0.03$ ) and $1.5 \pm 0.8 \mathrm{~mm}^{3}$ (14.7\% reduction); $2.5 \mathrm{mg} / \mathrm{kg}$ rapamycin: $3.1 \pm 0.9 \mathrm{~mm}^{3}$ (90.3\% reduction, $P<0.0001$ ), and $0.3 \pm 0.1 \mathrm{~mm}^{3}$ (82.9\% reduction, $\left.P=0.49\right)$; vehicle control: $42.1 \pm 5.5 \mathrm{~mm}^{3}$ and $1.7 \pm 0.7 \mathrm{~mm}^{3}$ ]. Histologic analysis of the residual limb and soft-tissue surrounding the AS with hematoxylin and eosin reinforced the findings of micro-CT and volumetric measurements (Figure 2A). At POD 84, mineralized and noncortical bone (woven bone) was observed in the soft tissue immediately surrounding the AS in injured rats administered vehicle control (Figure 2, $\mathrm{B}-\mathrm{D})$. In contrast, no measurable evidence of $\mathrm{HO}$ bone
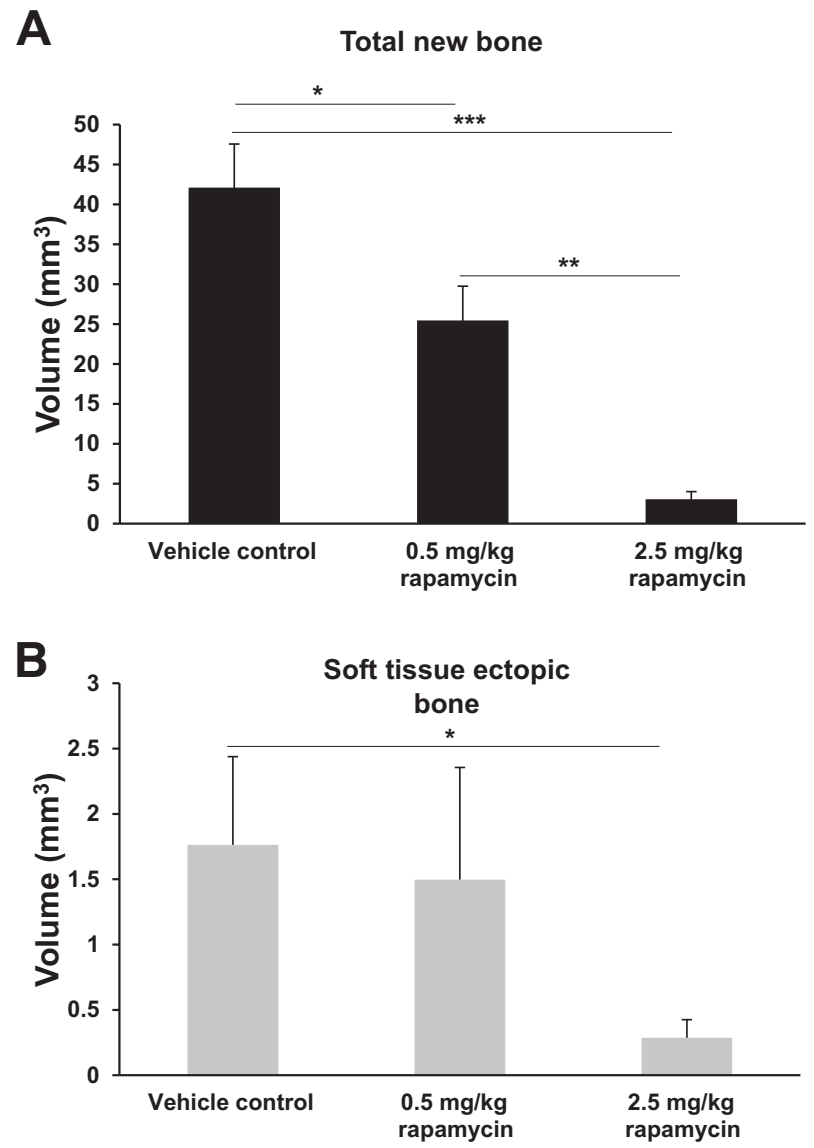

Figure 1 Quantitative analysis of heterotopic bone formation with and without rapamycin treatment. The total new bone (A) and soft-tissue ectopic bone (nonassociated with cortical margins; B) are quantified at postoperative day 84 . Vehicle control ( $3 \%$ dimethyl sulfoxide/saline). ${ }^{*} P<0.05,{ }^{* *} P<0.005$, and ${ }^{* *} P<0.0005$ (two-tailed $t$-test). 


\section{Vehicle control treated}
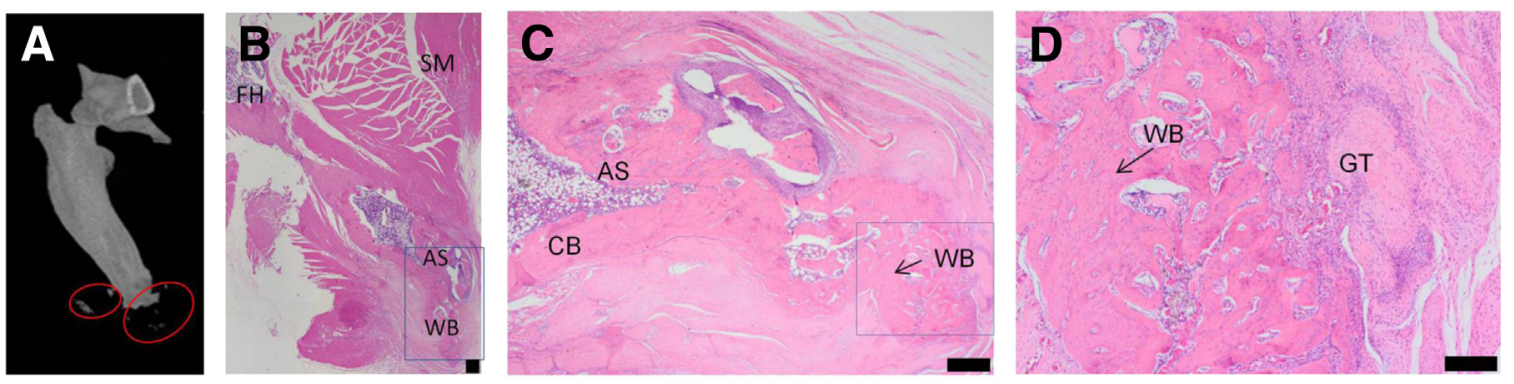

\section{Rapamycin treated}
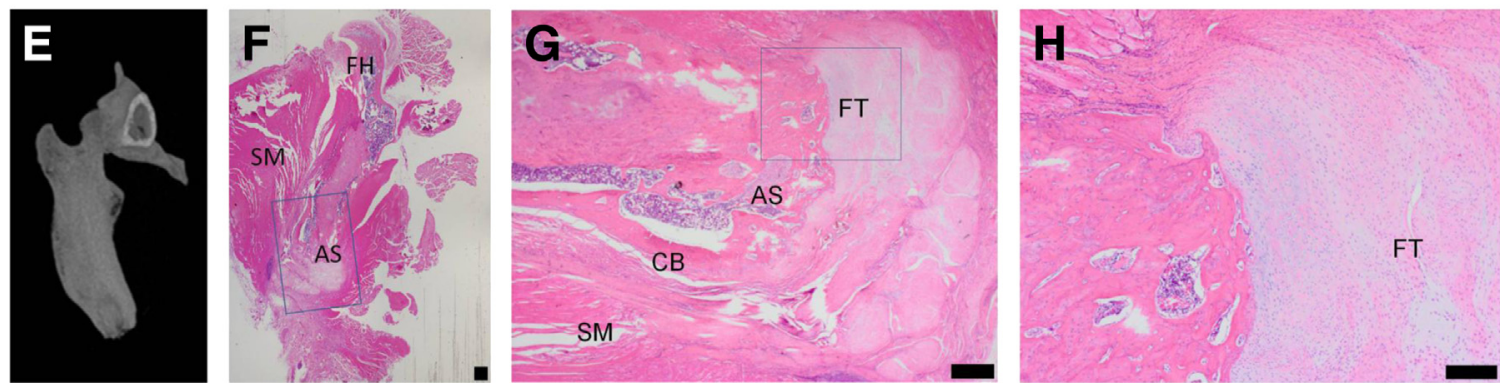

Figure 2 Rapamycin treatment attenuates the formation of ectopic bone in the blast-related extremity injury/methicillin-resistant Staphylococcus aureus infection model of heterotopic ossification ( $\mathrm{HO})$. A and E: Representative three-dimensional-rendered micro-computed tomography images of rat femurs at postoperative day 84 treated with vehicle control ( $3 \%$ dimethyl sulfoxide/saline; A) or rapamycin (2.5 mg/kg per day for 14 days, i.p; E). Areas of H0 formation (red circles). B-D and $\mathbf{F}-\mathbf{H}$ : Histologic assessment of hematoxylin and eosin-stained tissue sections from animals treated with vehicle control $(\mathbf{B}-\mathbf{D})$ and rapamycin $(\mathbf{F}-\mathbf{H})$. Areas of interest in near the distal end of the residual femur (boxed areas) in $\mathbf{B}, \mathbf{C}, \mathbf{F}$, and $\mathbf{G}$ were examined at higher magnification and are shown in C, D, G, and $\mathbf{H}$, respectively. Scale bars: $500 \mu \mathrm{m}(\mathbf{B}, \mathbf{C}, \mathbf{F}$, and $\mathbf{G}) ; 200 \mu \mathrm{m}(\mathbf{D}$ and $\mathbf{H})$. AS, amputation site; CB, cortical bone; FH, femoral head; FT, fibroblastic tissue; GT, granulation tissue; SM, skeletal muscle; WB, woven bone.

formation $\left(>0.05 \mathrm{~mm}^{3}\right)$ was noted radiographically or histologically in injured rats treated with rapamycin (Figure 2, E-H). Animals administered vehicle control demonstrated extensive regions of small-caliber blood vessels with large numbers of reactive fibroblasts, marking the granulation tissue, amid heterotopic woven bone and small patches of fibrous connective tissue. However, healing of the AS in the rapamycin-treated animals was accompanied by marked amount of fibrous connective tissue deposited at the site of amputation (fibroblastic tissue). There was no wound breakdown or infectious complications in either group.

Next, the potential antiangiogenic and antivascular effects of rapamycin treatment were examined on soft-tissue neovascularization by evaluating the distribution of neovessels using $\alpha$-SMA staining, a marker of smooth muscle cells. As expected, soft tissue around the zone of injury in rapamycintreated rats had a marked $69 \%$ reduction in the number of $\alpha-\mathrm{SMA}^{+}$vessels (vascular density) as seen by staining tissue sections with anti- $\alpha$-SMA (Figure $3 \mathrm{~A}$ ). Besides reductions in overall vascular density, a specific reduction in the size and staining intensity of $\alpha$-SMA ${ }^{+}$vessels was observed in tissues harvested from rapamycin-treated injured rats. Considering the well-documented role of expression of PDGFR $\alpha$ in muscle-resident progenitor cells, the localization of PDGFR $\alpha^{+}$cells was analyzed in the zone of injury on POD 7. A 52\% reduction in the number of
PDGFR $\alpha^{+}$cells was observed in rapamycin-treated rats after amputation relative to vehicle control-treated injured rats (Figure 3B).

Blast exposure plus extremity injury/amputation stimulates soft-tissue osteoprogenitor cell proliferation and expansion in the zone of injury. ${ }^{4}$ With the use of CTP colony-forming assays to assess progenitor cell frequency and proliferation, rapamycin treatment was found to decrease the number and proliferative activity of CTPs under both regular growth medium and osteogenic differentiation medium (CTP-Os) (Figure 4A). Further, the decline in osteogenic progenitor cell content and proliferative activity was accompanied by a twofold decrease in ALP activity $(P=0.004)$, a marker of early osteogenic differentiation (Figure 4B).

To examine whether critical signaling molecules shown to be involved in normal bone and ectopic bone formation can be down-modulated by rapamycin treatment, the expression level of mRNA transcripts for 83 target genes within muscle tissue harvested from the AS at POD 7 were analyzed. The genes comprise those involved in processes such as extracellular matrix synthesis, osteo-chondroadipogenesis, angiogenesis, injury, and inflammation. A 1000 -fold increase in expression of CD44 and a 500-fold increase in collagen 1a1 expression was observed in blastinjured animals treated with vehicle control compared 
A
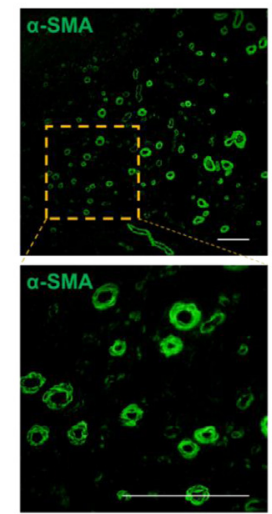

B
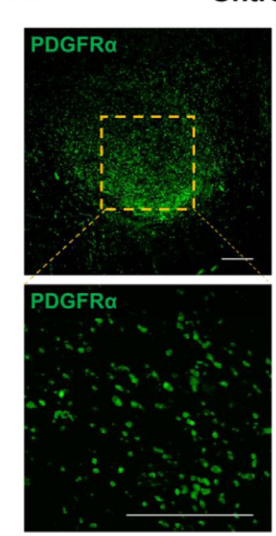

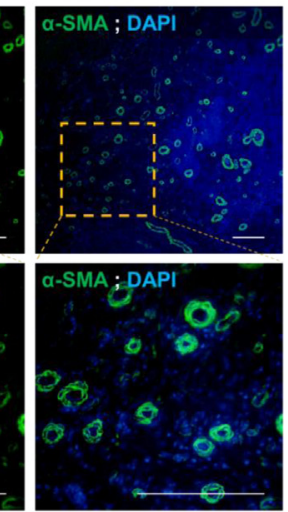

Untreated

Untreated
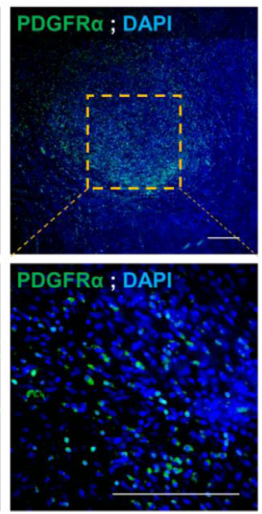
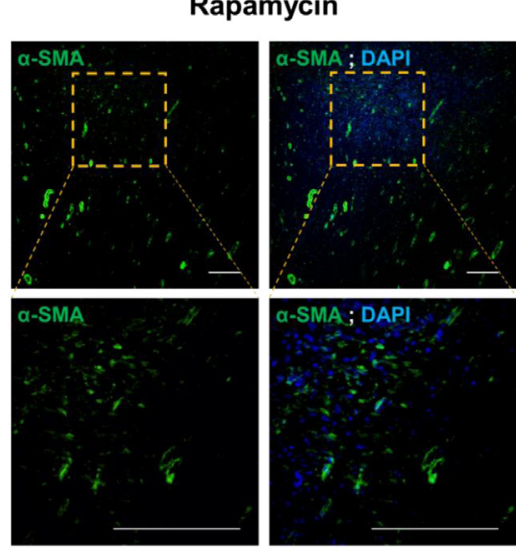

Rapamycin
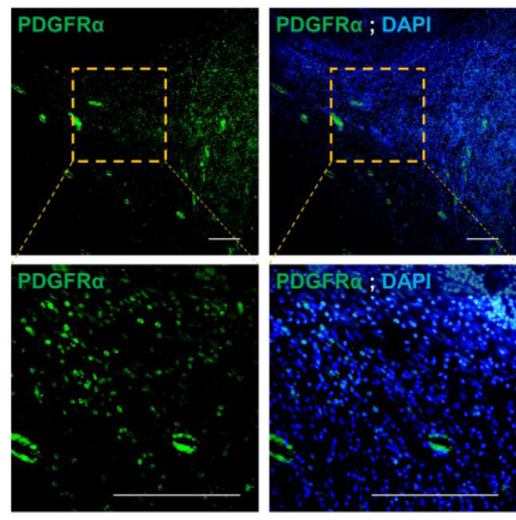

Injury site vascularity

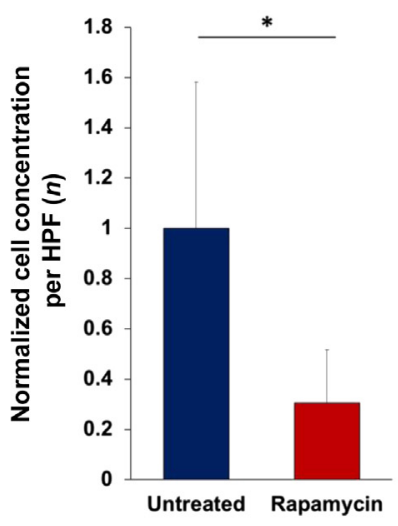

PDGFRa cellularity

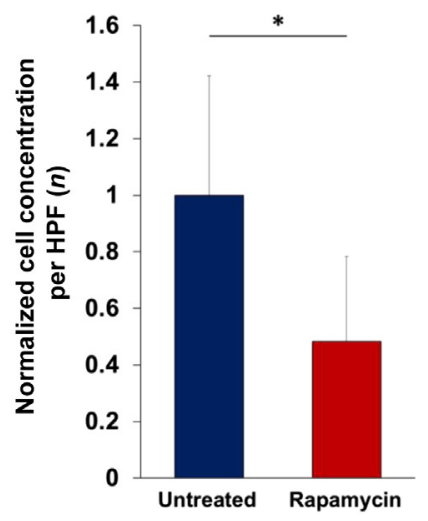

Figure 3 Effect of rapamycin on wound site vascularization/angiogenesis and platelet-derived growth factor receptor (PDGFR) $\alpha^{+}$osteogenic mesenchymal stromal cell progenitor cell content. Immunofluorescence staining of amputation site from naïve and rapamycin-treated animals for $\alpha$-smooth muscle actin $(\alpha-S M A ; A)$ and PDGFR $\alpha^{+}$(B) cells. DAPI marks cell nuclei. Data are expressed as the ratio of $\alpha$-SMA ${ }^{+}$vessels and PDGFR $\alpha^{+}$cells in rapamycin-treated versus untreated. ${ }^{*} P<0.05$ (two-tailed $t$-test). Scale bars $=100 \mu \mathrm{m}(\mathbf{A}$ and $\mathbf{B})$. HPF, high-powered field.

with naïve animals (Figure 5, A and B). CD44 is a membrane-bound receptor for hyaluronic acid, and like collagens, hyaluronic acid is abundantly expressed by chondrocytes. One week of rapamycin treatment resulted in down-regulation in the expression of these genes. Other extracellular matrix genes involved in endochondral ossification, such as integrin A (Itga), osteonectin (Sparc), and hyaluronan synthase (Has2), were also found to be significantly down-regulated in the rapamycin treatment group (Figure 5C). In addition, there was significant decrease in expression of early osteogenic transcription factors, such as Runx2, osterix ( $S p 7)$, and in the classic osteogenic ligand, Bmp2 (Figure 5D). We and others have previously reported up-regulation of inflammatory cytokines in the blast-injured animals at the site of injury, as early as 3 days after injury. ${ }^{8}$ Seven days after injury, although there was up-regulation of the chemokines Ccl2, Cxcl5, Cxcll1, and IL-6 in the vehicle-treated group, all these molecules were markedly down-regulated after rapamycin treatment (Figure 5E). There was also decrease in expression of the transforming growth factor $-\beta$ ligands, known to be involved in wound healing and fibrosis (Figure 5E). Muscle from rapamycintreated animals demonstrated significantly lower expression of the angiogenic marker, angiopoietin 2 (Angpt2) (Figure 5F) compared with the vehicle control, indicating toward rapamycin-induced inhibition of vasculogenesis. A panel of regulators of osteogenic differentiation (Scarb1, Tbx5, Twist1, Jag1, Ptchdl, and Smo) was also found to be significantly down-regulated in the rapamycin-treated group with respect to the vehicle control (Figure 5G). In addition, decrease in expression of adipogenic markers (Pparg and Adiporl) (Figure 5H) indicated toward an inhibitory effect of rapamycin on the multilineage differentiation potential of mesenchymal stem cells, with a reduction not only in osteogenic but also in adipogenic differentiation.

\section{Discussion}

The development of extraskeletal bone (HO) within damaged/healing tissue $\mathrm{e}^{1,18,19}$ is a significant complication of combat-related blast trauma and is described by clinicians as 

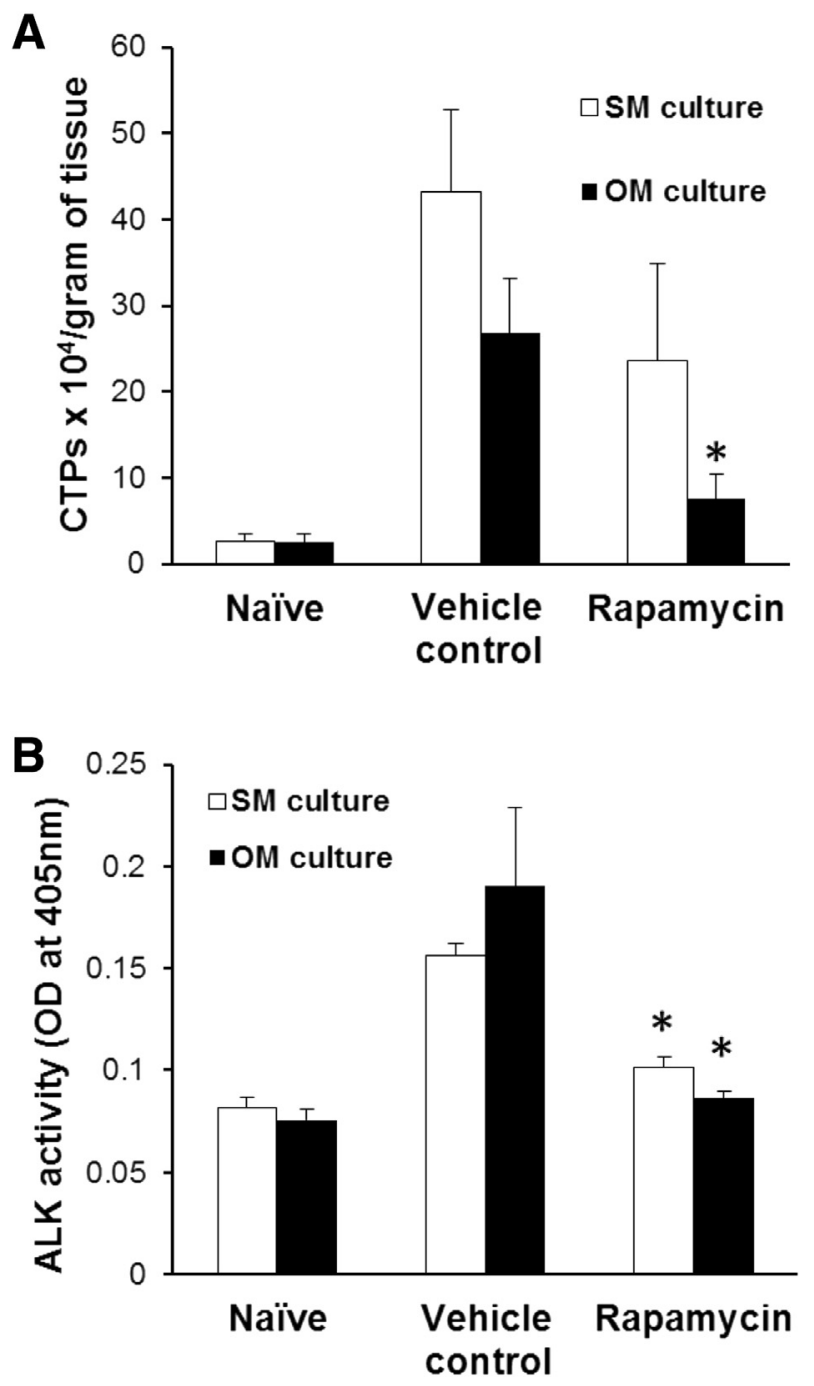

Figure 4 Alteration of connective tissue progenitor (CTP) frequency and osteogenic differentiation after rapamycin treatment. Shown is the frequency of CTPS in muscle tissue isolated from naïve, vehicle-, or rapamycin-treated injured rats (minus methicillin-resistant Staphylococcus aureus infection) at postoperative day 7, cultured either in growth (stromal) media (SM) or osteogenic media (OM) for 1 week (A) and early osteogenic differentiation of CTPS in SM or OM, as measured by p-nitrophenyl phosphate-based alkaline phosphatase activity assay (B). ${ }^{*} P<0.05$ versus vehicle control (two-tailed $t$-test).

the single most critical barrier to functional mobility, independence, and return to military service. ${ }^{20} \mathrm{HO}$ often leads to chronic pain, increased soft tissue breakdown, reduced limb and joint mobility, and/or impaired function of prosthetic limbs. Despite the exceptionally high rate of HO in combatinjured military personnel, there still remains no primary prophylactic regimen due to the logistical constraints of the forward deployed environment and medical contraindications. Strategies to disrupt the relevant osteoinductive pathways, alter the differentiation potential of endogeneous tissue-derived and/or recruited osteoprogenitor cells, and modify the osteoconductive microenvironment where $\mathrm{HO}$ forms are expected to prevent $\mathrm{HO} .^{21}$ Herein, we report clinically significant attenuation of HO (approximately $92 \%$ ) in our physiological rodent model of blast injuryextremity amputation, using a selective inhibitor of mTOR signaling, rapamycin.

The model used for this study is a well-established rat model developed in-house to closely recapitulate $\mathrm{HO}$ development after combat-related blast injury. ${ }^{22}$ Because this model encompasses all critical elements of a combat injury, namely long bone fracture, crush injury, wound infection, and lower extremity amputation, it serves as a pertinent model to test the efficacy of drugs targeting blast injury-related HO.

The mTOR signaling pathway nucleates multiple intracellular and extracellular signals within a cell, thereby serving as a key regulator of cell survival, proliferation, and metabolism. ${ }^{11}$ Rapamycin binds to FK506-binding protein 12 and this complex directly binds to mTOR, thereby inhibiting its function. ${ }^{11}$ With the use of transgenic mouse models, two recent studies elegantly demonstrated the role of mTOR signaling in promoting endochondral ossification. ${ }^{23,24}$ Although inhibition of one of the mTOR complexes, mTORC1, resulted in stalling of osteogenic differentiation at the early osteoblast stage, ${ }^{24,25}$ inhibition of mTORC2 resulted in aberrant chondrogenic hypertrophy, one of the critical early steps in endochondral ossification. ${ }^{23}$

The mTOR pathway is highly sensitive and responsive to changes in extracellular signals such as oxygen level, growth factors, nutrient concentrations, presence of cytokines, and other inflammatory molecules. ${ }^{11}$ A recent study using rapamycin demonstrated significant inhibition of trauma-induced and genetic $\mathrm{HO}^{26}$ in transgenic mouse models. This study used the burn injury-tenotomy trauma model, wherein a distant burn injury and tendon injury resulted in $\mathrm{HO}$ at the site of tenotomy. Treatment with rapamycin after burn-tenotomy injury resulted in significant attenuation of extraskeletal bone formation in this mouse model. In addition, knocking out hypoxia-inducible factor$1 \alpha$ in mesenchymal cells of the Prx lineage prevented HO, demonstrating the importance of hypoxic signaling in the phenomenon of $\mathrm{HO}^{26}$

The blast amputation extremity injury trauma model has multiple similarities with the burn-tenotomy model, in terms of activating a systemic immune response after trauma and extraskeletal ossification at the site of injury (AS and tenotomy site, respectively). Hence, the significant decrease in the volume of ectopic bone after rapamycin treatment observed in our study (Figure 1) closely corroborates with that seen in the burn-tenotomy model. ${ }^{26}$ Our model, however, is more relevant to combat and crush injuries, given the use of concomitant infection. Our model also allows a better assessment of wound healing, given the incidence of wound breakdown seen in MRSA-treated animals. ${ }^{7}$ Histologic analysis of the AS showed complete absence of hypertrophic chondrocytes in the rapamycin-treated animals (Figure 2). Regions of active chondroblast proliferation and differentiation (chondrogenesis) were delayed and reduced 

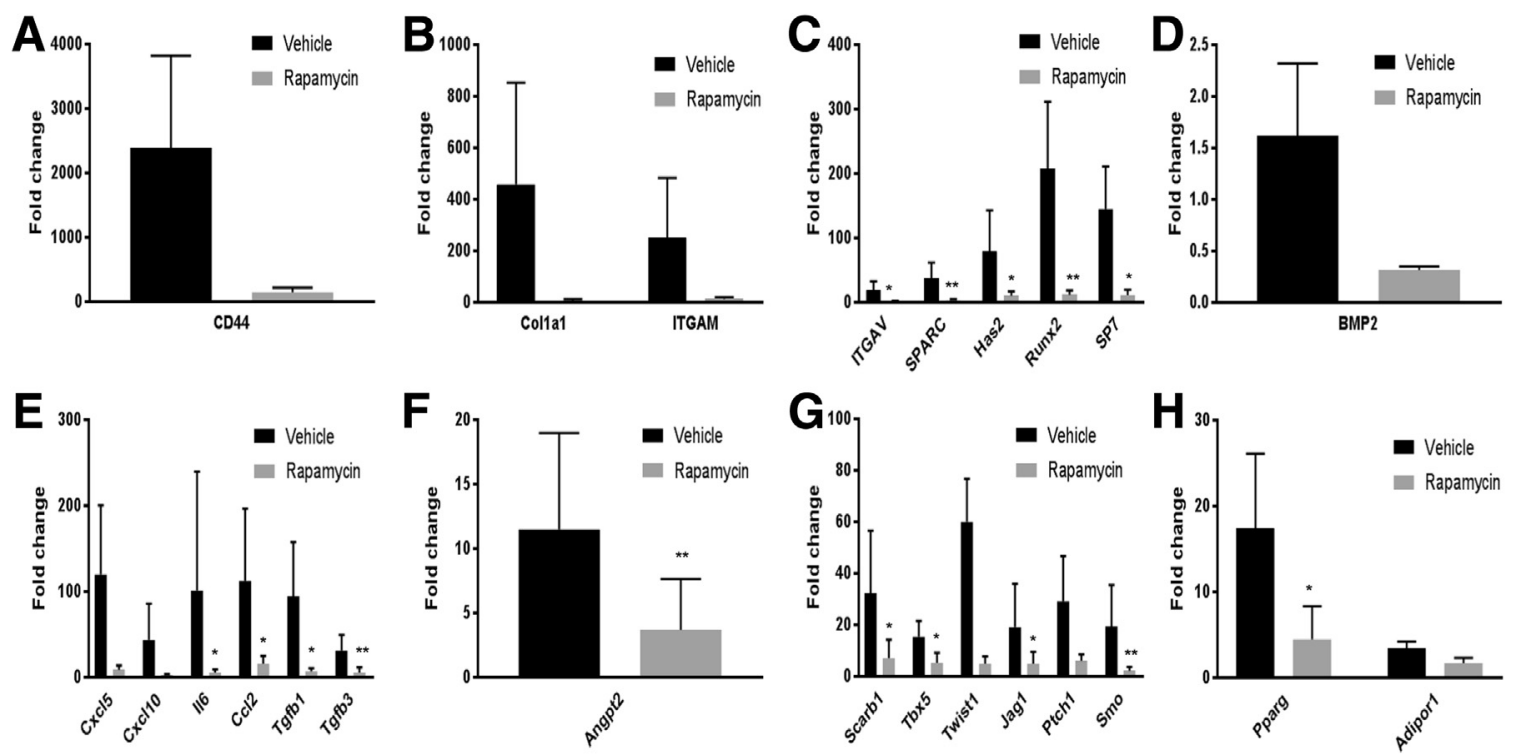

Figure 5 Effects of rapamycin treatment and vehicle control on expression of extracellular matrix and osteogenic genes (A-D), inflammatory cytokines $(\mathbf{E})$, angiopoietin $(\mathbf{F})$, osteogenic regulators $(\mathbf{G})$, and adipogenic genes $(\mathbf{H})$. The fold-change in gene expression was calculated by normalizing the expression values to those from noninjured, naïve muscle tissue. $n=4$ rapamycin-treated $(\mathbf{A}-\mathbf{H}) ; n=4$ vehicle control $(\mathbf{A}-\mathbf{H})$. ${ }^{*} P<0.05,{ }^{* *} P<0.005$ versus vehicle control (analysis of variance, followed by Tukey's test).

in rapamycin-treated rats, wherein immature cartilaginous extracellular matrix was replaced by FT, rather than ectopic endochondral bone. Given that hypoxia is essential for chondrogenic differentiation, ${ }^{26}$ this might be one of the mechanisms by which rapamycin inhibits ectopic bone formation in our model.

The decrease in endochondral differentiation seen in rapamycin-treated animals is corroborated by our gene expression results in which there was significant downregulation of osteogenic ligands, transcription factors, (Bmp2, Sp7, Runx2), and pro-osteogenic ECM-associated genes (Cd44, Collal, Has2, integrins) as early as 1 week after rapamycin treatment. Although collagen 1 is known as a critical ECM component of mature bone, hyaluronan synthase (Has2) gene encodes hyaluronic acid, extensively found associated with hypertrophic chondrocytes, ${ }^{27}$ and CD44 is the receptor of hyaluronic acid. ${ }^{28}$

Our results also demonstrate the antiangiogenic effect of rapamycin, as indicated by the marked decrease in the frequency of $\alpha$-SMA-expressing blood vessels, PDGFR $\alpha$ expression at the AS (Figure 3), and down-regulation in the expression of angiopoietin 2 gene (Figure 5E). Vascularization of the cartilaginous matrix is key for osteogenesis during the process of endochondral ossification. Hypoxiadriven hypoxia-inducible factor- $1 \alpha$ signaling within the chondrocytic growth plate plays a critical role in neoangiogenesis of the developing bone. ${ }^{29}$ Impaired chondrogenic differentiation at the AS in the rapamycin-treated rats appears to preclude neovascularization, and therefore osteoblast homing and differentiation at the site of injury. It is important to note, however, that even with its inhibitory angiogenic role, rapamycin had no deleterious effect on the rate of wound closure and wound dehiscence as we have seen with other treatments. ${ }^{7}$

Blast injury and extremity amputation results in a large increase in the frequency of colony-forming CTPs within injured soft tissue (Figure 4A). Injury appears to trigger proliferation of CTPs in both growth and osteogenic differentiation media. Rapamycin treatment resulted in marked reduction in the frequency of this cell population, as early as 7 days after treatment. Antiproliferative effects of rapamycin has been reported in a variety of cell types, ${ }^{30-32}$ including a recent report that demonstrated the inhibitory effect of rapamycin on bone morphogenetic protein 2-mediated proliferation of carcinoma cells. ${ }^{33}$ In all these studies, rapamycin inhibited proliferation by the mTORC1 complex.

CTPs isolated after 1 week of rapamycin treatment also demonstrated marked reduction in early osteogenic differentiation (Figure 4B). ALP activity is detected at the highest levels during the early stages of endochondral ossification, typically in hypertrophic chondrocytes and early osteoblasts. ${ }^{34,35}$ As noted earlier, mTORC1 and mTORC2 complexes are essential in development and function of pre-osteoblasts and hypertrophic chondrocytes, respectively. ${ }^{23,24}$ Hence, rapamycin might inhibit HO by blocking these early steps of endochondral ossification within O-CTP cells.

Our trauma model involves extensive local and systemic inflammation, ${ }^{36}$ resulting in highly complex interactions between resident cells, soluble mediators, and recruited/ mobilized cells such as neutrophils, macrophages, endothelial progenitor cells, and multipotent mesenchymal stromal cells. This is clearly observed in the histologic analysis of the AS (Figure 2), extensive areas of granulation tissue 
can be seen, marked by large number of reactive fibroblasts, which typically represents incomplete (or immature) wound healing. In contrast, the injury at the AS in rapamycintreated animals showed complete healing, with a well-defined fibrous tissue and absence of reactive fibroblasts. As a well-established immunosuppressant, systemic administration of rapamycin might effectively inhibit homing of osteogenic precursors to the AS, resulting in the observed decrease in frequency of O-CTP cells. However, inflammation is also known to suppress skeletal muscle regeneration, ${ }^{37}$ which in turn is reversed by rapamycin treatment. In fact, systemic administration of rapamycin was shown to prevent vascular smooth muscle cell proliferation and re-endothelialization due to reduced adhesion of circulating $\mathrm{CD}_{4} 5^{+}$and bone-marrow-derived $\mathrm{CD} 34^{+}$progenitors to activated endothelial cells by reduced levels of adhesion molecules (VCAMI and ICAMI). ${ }^{38}$ After rapamycin treatment, a $>1000$-fold reduction in gene expression of the adhesion molecule $C D 44$, significant reduction in integrin- $\alpha$ (Figure 5, A and B), and multiple chemokines and cytokines, namely $\mathrm{Cxcl5}, \mathrm{Cxcl10}$, IL6, and Ccl2 (Figure 5D), was also observed.

Overall, in our blast injury-extremity amputation model, systemic administration of rapamycin was seen to inhibit $\mathrm{HO}$ at the injury site by a multipronged approach, involving reduction in inflammation, angiogenesis, and reduced frequency of osteogenic progenitors within the injury site. More importantly, no rebound effect was seen after cessation of treatment. These findings indicate that mTORdependent inhibition is a viable therapeutic option to prevent the development of blast trauma-induced HO. Going further, it would be interesting to analyze the specific cell types inhibited systemically and at the site of injury by rapamycin treatment. Given these and the fact that rapamycin is an FDA-approved drug (sirolimus), makes it a promising candidate for fast-track translation to the clinic.

There are some notable limitations in our present study. First, as previously established, the formation of ectopic bone in our rat model follows a classic endochondral ossification process, ${ }^{8}$ the same phenomenon underlying fracture healing. Considering the relatively high prevalence of fracture and wound-related complications in the combat injured, definitive strategies for fracture fixation, wound management, and soft tissue reconstruction need to be addressed simultaneously while stabilizing and treating $\mathrm{HO}$ in these patients. Given the striking similarities between fracture healing and endochondral ectopic bone formation, the inhibitory effect of rapamycin on fracture healing and its dosing schemes need to be outlined for an acceptable translation of the drug into a clinical setting. Thus, early detection of patients at high risk of $\mathrm{HO}$ and brief timed administration might be required to maximize HO prophylaxis and to minimize fracture nonunion. Second, rapamycin is a potent immunosuppressant, widely used as an antirejection treatment for prevention of allograft rejection after renal transplantation. ${ }^{13}$ In our study, we did not quantify the levels of systemic and local immune-regulatory markers or the influence of rapamycin treatment on acute and persistent infection resulting from the inoculation with MRSA. ${ }^{17}$ It is imperative that the profound attenuation of $\mathrm{HO}$ attained with rapamycin in our trauma model is not accompanied with a compromised immune system which can potentially hinder the effectiveness of a variety of clinical strategies used to control and treat persistent infection arising from foreign debris and contaminants in the clinical combatinjured wounds.

There is an unmet clinical need for an effective, tolerable, and long-lasting therapeutic strategy to prevent $\mathrm{HO}$ after trauma-induced injury. The data generated from this study established the efficacy and safety of an FDA-approved drug, rapamycin, for the prevention of trauma-induced HO. However, additional preclinical evidence is necessary to assess timing, potential impact on fracture healing and immune response, and after healing functional improvement for successful translation of the drug into a clinical setting.

\section{Acknowledgment}

We thank the Naval Medical Research Center's Department of Pathology personnel with special thanks to MAJ. Margaret A. Hanson.

\section{References}

1. Potter BK, Burns TC, Lacap AP, Granville RR, Gajewski DA: Heterotopic ossification following traumatic and combat-related amputations. Prevalence, risk factors, and preliminary results of excision. J Bone Joint Surg Am 2007, 89:476-486

2. Forsberg JA, Pepek JM, Wagner S, Wilson K, Flint J, Andersen RC Tadaki D, Gage FA, Stojadinovic A, Elster EA: Heterotopic ossification in high-energy wartime extremity injuries: prevalence and risk factors. J Bone Joint Surg Am 2009, 91:1084-1091

3. Tintle SM, Shawen SB, Forsberg JA, Gajewski DA, Keeling JJ, Andersen RC, Potter BK: Reoperation after combat-related major lower extremity amputations. J Orthop Trauma 2014, 28:232-237

4. Davis TA, O'Brien FP, Anam K, Grijalva S, Potter BK, Elster EA: Heterotopic ossification in complex orthopaedic combat wounds: quantification and characterization of osteogenic precursor cell activity in traumatized muscle. J Bone Joint Surg Am 2011, 93:1122-1131

5. Gordon W, Kuhn K, Staeheli G, Dromsky D: Challenges in definitive fracture management of blast injuries. Curr Rev Musculoskelet Med 2015, 8:290-297

6. Seavey JG, Wheatley BM, Pavey GJ, Tomasino AM, Hanson MA, Sanders EM, Dey D, Moss KL, Potter BK, Forsberg JA, Qureshi AT, Davis TA: Early local delivery of vancomycin suppresses ectopic bone formation in a rat model of trauma-induced heterotopic ossification. J Orthop Res 2017, [Epub ahead of print] doi:10.1002/jor.23544

7. Pavey GJ, Qureshi AT, Tomasino AM, Honnold CL, Bishop DK, Agarwal S, Loder S, Levi B, Pacifici M, Iwamoto M, Potter BK, Davis TA, Forsberg JA: Targeted stimulation of retinoic acid receptorgamma mitigates the formation of heterotopic ossification in an established blast-related traumatic injury model. Bone 2016, 90: $159-167$

8. Qureshi AT, Crump EK, Pavey GJ, Hope DN, Forsberg JA, Davis TA: Early characterization of blast-related heterotopic ossification in a rat model. Clin Orthop Relat Res 2015, 473:2831-2839 
9. Davis TA, Lazdun Y, Potter BK, Forsberg JA: Ectopic bone formation in severely combat-injured orthopedic patients - a hematopoietic niche. Bone 2013, 56:119-126

10. Hadji P, Coleman R, Gnant M: Bone effects of mammalian target of rapamycin (mTOR) inhibition with everolimus. Crit Rev Oncol Hematol 2013, 87:101-111

11. Laplante M, Sabatini DM: mTOR signaling at a glance. J Cell Sci 2009, 122:3589-3594

12. Laplante M, Sabatini DM: mTOR signaling in growth control and disease. Cell 2012, 149:274-293

13. Morath C, Arns W, Schwenger V, Mehrabi A, Fonouni H, Schmidt J, Zeier M: Sirolimus in renal transplantation. Nephrol Dial Transplant 2007, 22 Suppl 8:viii61-viii65

14. Harasawa M, Yasuda M, Hirasawa T, Miyazawa M, Shida M, Muramatsu T, Douguchi K, Matsui N, Takekoshi S, Kajiwara H, Yoshiyuki Osamura R, Mikami M: Analysis of mTOR inhibitioninvolved pathway in ovarian clear cell adenocarcinoma. Acta Histochem Cytochem 2011, 44:113-118

15. Chen J, Zheng XF, Brown EJ, Schreiber SL: Identification of an 11$\mathrm{kDa}$ FKBP12-rapamycin-binding domain within the 289-kDa FKBP12-rapamycin-associated protein and characterization of a critical serine residue. Proc Natl Acad Sci U S A 1995, 92:4947-4951

16. Hudson CC, Liu M, Chiang GG, Otterness DM, Loomis DC, Kaper F, Giaccia AJ, Abraham RT: Regulation of hypoxia-inducible factor 1alpha expression and function by the mammalian target of rapamycin. Mol Cell Biol 2002, 22:7004-7014

17. Pavey GJ, Qureshi AT, Hope DN, Pavlicek RL, Potter BK, Forsberg JA, Davis TA: Bioburden increases heterotopic ossification formation in an established rat model. Clin Orthop Relat Res 2015, 473:2840-2847

18. Forsberg JA, Potter BK: Heterotopic ossification in wartime wounds. J Surg Orthop Adv 2010, 19:54-61

19. Potter BK, Burns TC, Lacap AP, Granville RR, Gajewski D: Heterotopic ossification in the residual limbs of traumatic and combat-related amputees. J Am Acad Orthop Surg 2006, 14:S191-S197

20. Alfieri KA, Forsberg JA, Potter BK: Blast injuries and heterotopic ossification. Bone Joint Res 2012, 1:192-197

21. Kaplan FS, Glaser DL, Hebela N, Shore EM: Heterotopic ossification. J Am Acad Orthop Surg 2004, 12:116-125

22. Polfer EM, Hope DN, Elster EA, Qureshi AT, Davis TA, Golden D, Potter BK, Forsberg JA: The development of a rat model to investigate the formation of blast-related post-traumatic heterotopic ossification. Bone Joint J 2015, 97-B:572-576

23. Chen J, Holguin N, Shi Y, Silva MJ, Long F: mTORC2 signaling promotes skeletal growth and bone formation in mice. J Bone Miner Res 2015, 30:369-378

24. Fitter S, Matthews MP, Martin SK, Xie J, Ooi SS, Walkley CR, Codrington JD, Ruegg MA, Hall MN, Proud CG, Gronthos S, Zannettino AC: mTORC1 plays an important role in skeletal development by controlling preosteoblast differentiation. Mol Cell Biol 2017, 37:e00668-16

25. Li SF, Tang JJ, Chen J, Zhang P, Wang T, Chen TY, Yan B, Huang B, Wang L, Huang MJ, Zhang ZM, Jin DD: Regulation of bone formation by baicalein via the mTORC1 pathway. Drug Des Devel Ther 2015, 9: $5169-5183$

26. Agarwal S, Loder S, Brownley C, Cholok D, Mangiavini L, Li J, Breuler C, Sung HH, Li S, Ranganathan K, Peterson J, Tompkins R, Herndon D, Xiao W, Jumlongras D, Olsen BR, Davis TA, Mishina Y, Schipani E, Levi B: Inhibition of Hif1alpha prevents both traumainduced and genetic heterotopic ossification. Proc Natl Acad Sci U S A 2016, 113:E338-E347

27. Muzzarelli RA, Greco F, Busilacchi A, Sollazzo V, Gigante A: Chitosan, hyaluronan and chondroitin sulfate in tissue engineering for cartilage regeneration: a review. Carbohydr Polym 2012, 89:723-739

28. Altman RD, Manjoo A, Fierlinger A, Niazi F, Nicholls M: The mechanism of action for hyaluronic acid treatment in the osteoarthritic knee: a systematic review. BMC Musculoskelet Disord 2015, 16:321

29. Maes C, Carmeliet P, Moermans K, Stockmans I, Smets N, Collen D, Bouillon R, Carmeliet G: Impaired angiogenesis and endochondral bone formation in mice lacking the vascular endothelial growth factor isoforms VEGF164 and VEGF188. Mech Dev 2002, 111:61-73

30. Liu L, Pan Y, Song Y, Su X, Ke R, Yang L, Gao L, Li M: Activation of AMPK alpha2 inhibits airway smooth muscle cells proliferation. Eur J Pharmacol 2016, 791:235-243

31. Zeng Q, Qin S, Zhang H, Liu B, Qin J, Wang X, Zhang R, Liu C, Dong X, Zhang S, Huang S, Chen L: Rapamycin attenuates BAFFextended proliferation and survival via disruption of mTORC1/2 signaling in normal and neoplastic B-lymphoid cells. J Cell Physiol 2017, [Epub ahead of print] doi:10.1002/jcp.25913

32. Wang M, Guo Y, Wang M, Zhou T, Xue Y, Du G, Wei X, Wang J, Qi L, Zhang H, Li L, Ye L, Guo X, Wu X: The glial cell-derived neurotrophic factor (GDNF)-responsive phosphoprotein landscape identifies raptor phosphorylation required for spermatogonial progenitor cell proliferation. Mol Cell Proteomics 2017, 16:982-997

33. Wang MH, Zhou XM, Zhang MY, Shi L, Xiao RW, Zeng LS, Yang XZ, Zheng XFS, Wang HY, Mai SJ: BMP2 promotes proliferation and invasion of nasopharyngeal carcinoma cells via mTORC1 pathway. Aging (Albany NY) 2017, 9:1326-1340

34. Stephens M, Kwan AP, Bayliss MT, Archer CW: Human articular surface chondrocytes initiate alkaline phosphatase and type X collagen synthesis in suspension culture. J Cell Sci 1992, 103(Pt 4):1111-1116

35. Bahrami S, Plate U, Dreier R, DuChesne A, Willital GH, Bruckner P: Endochondral ossification of costal cartilage is arrested after chondrocytes have reached hypertrophic stage of late differentiation. Matrix Biol 2001, 19:707-715

36. Forsberg JA, Potter BK, Polfer EM, Safford SD, Elster EA: Do inflammatory markers portend heterotopic ossification and wound failure in combat wounds? Clin Orthop Relat Res 2014, 472:2845-2854

37. White JP, Puppa MJ, Gao S, Sato S, Welle SL, Carson JA: Muscle mTORC1 suppression by IL-6 during cancer cachexia: a role for AMPK. Am J Physiol Endocrinol Metab 2013, 304: E1042-E1052

38. Daniel JM, Dutzmann J, Brunsch H, Bauersachs J, Braun-Dullaeus R, Sedding DG: Systemic application of sirolimus prevents neointima formation not via a direct anti-proliferative effect but via its antiinflammatory properties. Int J Cardiol 2017, 238:79-91 